\title{
Digitale Ästhetik: CAD/CAM-Material der nächsten Generation
}

\author{
Vermehrt setzen sich digitale Verfahren in der Zahnmedizin durch. So haben in den letzten Jahr- \\ zehnten unter anderem CAD/CAM-Systeme Einzug in die Behandlungskette gehalten und er- \\ möglichen neue Therapieformen: Chairside können Patienten beispielsweise in 1 Sitzung mit \\ bestimmten indirekten Restaurationen versorgt werden. Die Fertigung der benötigten Werk- \\ stücke erfolgt dabei aus CAD/CAM-Blöcken mithilfe von Fräs- und Schleifeinheiten vor Ort.
}

Die Qualität der Versorgungen wird wesentlich vom verwendeten Material bestimmt - hier setzt die speziell entwickelte Hybridkeramik CERASMART (GC) neue Maßstäbe: Sie kombiniert die Vorzüge von 2 bewährten Werkstoffen und liefert bei Einzelzahnrestaurationen hochästhetische Ergebnisse. Neben der Herstellung von Inlays, Onlays und Veneers sind die Blöcke im Gegensatz zu vergleichbaren Materialien auch zur Fertigung von Kronen geeignet, da sie aufgrund ihrer Ätzbarkeit über einen sehr guten Haftverbund verfügen.

Die Praxis wird digital. Seit der Einführung von CEREC vor 30 Jahren hat sich die Chairside-Fertigung von Zahnersatz ständig weiterentwickelt. Heute sind digitale Verfahren nicht mehr aus modernen Zahnarztpraxen wegzudenken. Die Prozesskette reicht von der Erfassung der Mundsituation und des Modells - mithilfe von Intraund Extraoralscannern - über das virtuelle Design bis hin zur Fertigung der prothetischen Arbeit. Hier sind trotz des Trends zu additiven Verfahren abtragende Fertigungsmethoden State of the Art.

Effiziente Fräs- und Schleifeinheiten für die Praxis fügen sich hierbei perfekt in den Behandlungsfluss ein und der digitale Workflow gewährleistet eine effiziente Patientenversorgung - bei gleichzeitig hoher Qualität des Zahnersatzes. So ist eine Auslagerung der Fertigung zumindest bei einfachen Einzelzahnrestaurationen nun meist überflüssig. Vollkeramische Inlays lassen sich beispielsweise innerhalb einer einzigen Behandlungssitzung fertigen und eingliedern. Für Einzelzahn-Restaurationen hat sich diese Chairside-Variante daher als langzeitstabile und wirtschaftliche Versorgungsform durchgesetzt (Wittneben JG et al. Int J Prosthodont 2009; 22: 466-471).
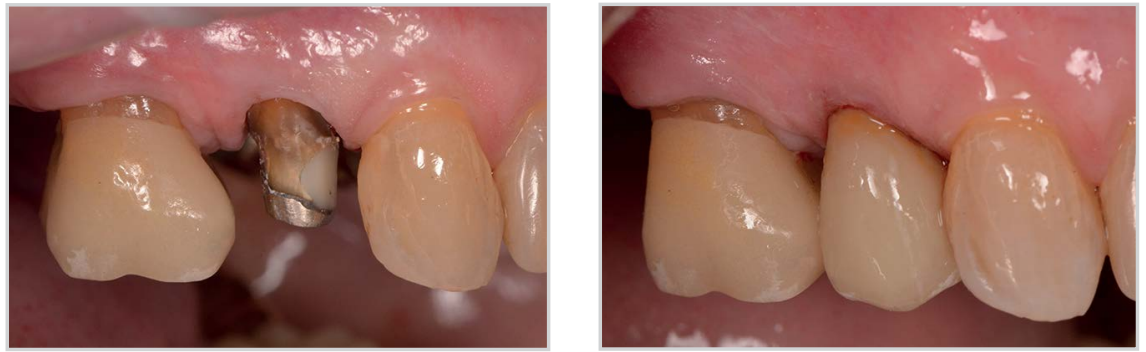

Abb. 1 a, b Vorher-Nachher-Aufnahme einer Kronenrestauration mit CERASMART (Quelle: Prof. Marleen Peumans, Belgien).

Auch die Restaurationsmaterialen haben sich im Laufe der Zeit gewandelt. Früher im Bereich der Prothetik noch weit verbreitet, ist beispielsweise die Versorgung mit Gold aufgrund gestiegener Rohstoffpreise und der unnatürlichen Optik oftmals nicht mehr erwünscht; der Patient verlangt heute nach zahnfarbenen Zahnersatz. Deshalb sind alternative Werkstoffe besonders gefragt und werden in $\mathrm{Zu}$ kunft noch an Bedeutung gewinnen. Die digitalen Verarbeitungstechniken sind dabei besonders interessant, weil sie es ermöglichen, Materialien $\mathrm{zu}$ verarbeiten, die zuvor nicht im Fokus standen - wie beispielsweise Zirkon. Eine relativ neuartige Werkstoffklasse innerhalb der Keramiken bilden Hybridkeramiken wie CERASMART von GC. Das japanische Unternehmen bietet neben einer Universal-Variante des Materials auch eine für die Bearbeitung mit CEREC-Schleifmaschinen konzipierte
Version an, die damit prädestiniert für den Chairside-Einsatz in der Praxis ist. Beide sind geeignet für die Herstellung von metallfreien, indirekten Restaurationen wie Inlays, Onlays, Veneers und auch

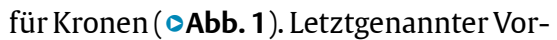
teil ist darauf zurückzuführen, dass CERASMART-Blöcke ätzbar sind und so über einen sehr guten Haftverbund verfügen. 

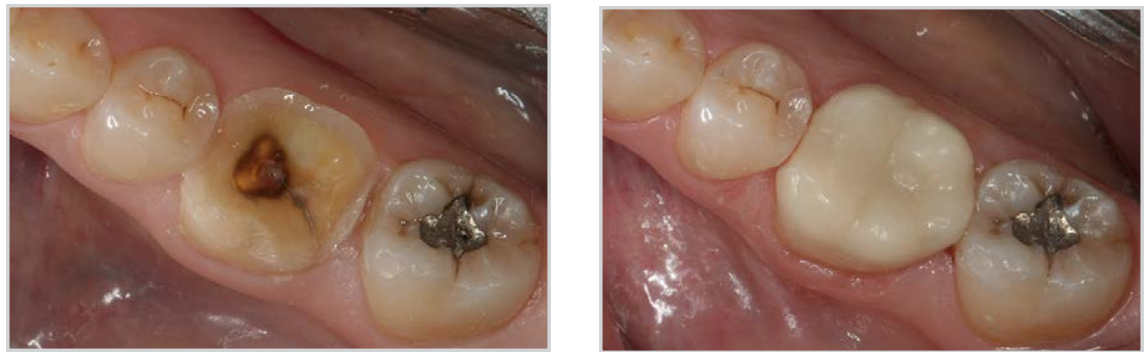

Abb. 3 a, b Vorher-/Nachher-Aufnahme eines Hybridkeramik-Inlays (CERASMARTTM, GC) (Quelle: Dr. Rich Rosenblatt, USA).

\section{Hybridpower für den (digitalen) Praxis-Workflow}

Aus der Automobilindustrie ist der Begriff Hybrid seit geraumer Zeit bekannt. Hier werden beispielsweise Antriebstechniken wie Benzin- und Elektromotor - die für sich schon sehr gut funktionieren - kombiniert, um das Fahrzeug noch effizienter fortzubewegen. Ein ähnliches Prinzip liegt den Hybridkeramiken zugrunde. Diese haben den Anspruch, die positiven Eigenschaften der bewährten Material-Lösungen Keramik und Komposit zu kombinieren und einen neuen optimierten Werkstoff zu bilden, um eine kostengünstige und gleichzeitig qualitativ hochwertige Versorgung der Patienten sicherzustellen. CERASMART setzt dabei auf modernste Nanofüllertechnologie: Extrem feine (300 $\mathrm{nm}$ ), homogen verteilte Füller sorgen für einen langanhaltenden Glanz und eine geringe Abnutzung der Antagonisten des Gegenkiefers. Darüber hinaus bietet es im Vergleich mit Materialien von Wettbewerbern wie beispielsweise Lava Ultimate (3M ESPE, Seefeld) und Enamic (Vita Zahnfabrik, Bad Säckingen) nicht nur die höchste Biegefestigkeit, sondern auch die höchsten Flexibilität ( $\triangle$ Abb. 2) zur Abdämpfung des Kaudrucks, weshalb es sich besonders gut beispielsweise zur Versor- gung von Patienten mit Bruxismus eignet. Die optimierten Eigenschaften von CERASMART haben auch direkten Einfluss auf den digitalen Workflow in der Praxis. Der Werkstoff kann aufgrund seiner Materialstruktur besonders effizient gefräst werden und ermöglicht damit kürzere Bearbeitungszeiten. So lässt sich die virtuelle Situation besonders schnell umsetzen. Gleichzeitig nutzen sich die Fräs- und Schleifwerkzeuge bei der Bearbeitung weniger ab, was deren längere Haltbarkeit begünstigt.

\section{Ästhetische CAD/CAM-Versorgung $\nabla$}

Auch bei der optischen Veredelung ist die Hybridkeramik besonders komfortabel handzuhaben. Sie besticht mit einer ausgezeichneten Polierbarkeit; so erhält der Anwender schon nach kurzer Zeit ein glänzendes Ergebnis - zum Beispiel mit der Diamant-Polierpaste DiaPolisher Paste (GC). Im Gegensatz zu konventionellen Keramiken benötigt das Material keinen Brennofen, sondern kann schnell, bequem und ohne zusätzliche Geräteinvestitionen charakterisiert werden. Denn mit der lichthärtenden Oberflächenversiegelung Optiglaze color (GC) - die für Glanz, Farbgebung und Schutz der Restauration sorgt
- gestaltet sich die Charakterisierung äußerst komfortabel. In Verbindung mit der ausbalancierten Fluoreszenz und Opaleszenz des Materials sorgt der Anwender dafür, dass die prothetische Arbeit beinahe unsichtbar wird und erfüllt so Chairside den Patientenwunsch nach einer möglichst ästhetischen, zahnfarbenen Versorgung (๑Abb. 3).

\section{CERASMART - Wirtschaftlichkeit, Effizienz und Ästhetik}

Digitale Fertigungsverfahren eröffnen dem Zahnarzt eine Vielzahl an neuen Möglichkeiten. Dabei nimmt mit der steigenden Qualität der angebotenen Materialen auch die Qualität der Versorgung zu. Moderne Hybridkeramiken wie CERASMART haben direkten positiven Einfluss auf den Workflow in der Praxis. Sie überzeugen durch eine schnelle und präzise Fräsbarkeit. Darüber hinaus besticht CERASMART aufgrund seiner Hybridstruktur durch Stabilität und Flexibilität, was eine Eignung für Implantatkronen und den Wegfall von Chipping nach sich zieht. Gleichzeitig genügt es höchsten ästhetischen Ansprüchen. Die ChairsideVersorgung mit modernen CAD/CAM-Materialen wie die Hybridkeramik ist deshalb längst mehr als eine Behandlungsalternative bei Einzelzahnrestaurationen. Sie verbindet Wirtschaftlichkeit, Effizienz und Ästhetik und eröffnet so eine glänzende Zukunft für den behandelnden Arzt und die Patienten.

\section{Kontakt}

GC Germany GmbH

Seifgrundstraße 2

61348 Bad Homburg

www.germany.gceurope.com

info@germany.gceurope.com 